\title{
Origin of the X-ray off-states in Vela X-1
}

\author{
A. Manousakis ${ }^{1,2,3}$ and R. Walter ${ }^{2,3}$ \\ ${ }^{1}$ Centrum Astronomiczne im. M. Kopernika, Bartycka 18, 00716 Warszawa, Poland \\ e-mail: antonism@camk.edu.pl \\ 2 ISDC Data Center for Astrophysics, Université de Genève, Chemin d'Ecogia 16, 1290 Versoix, Switzerland \\ 3 Observatoire de Genève, Université de Genève, Chemin des Maillettes 51, 1290 Versoix, Switzerland
}

Received 5 March 2013 / Accepted 17 December 2014

\section{ABSTRACT}

\begin{abstract}
Context. Vela X-1 is the prototype of the classical super-giant high mass X-ray binary systems. Recent continuous and long monitoring campaigns revealed a large hard X-rays variability amplitude with strong flares and off-states. This activity has been interpreted by invoking clumpy stellar winds and/or magnetic gating mechanisms.

Aims. We investigate whether the observed behavior may be explained by unstable hydrodynamic flows close to the neutron star instead of by the more exotic phenomena.

Methods. We used the hydrodynamic code VH-1 to simulate the flow of the stellar wind with high temporal resolution and to compare the predicted accretion rate with the observed light curves.

Results. The simulation results are similar to the observed variability. Off-states are predicted with a duration of 5 to 120 min corresponding to transient low-density bubbles forming around the neutron star. Oscillations of the accretion rate with a typical period of $\sim 6800 \mathrm{~s}$ are generated in our simulations and observed. They correspond to the complex motion of a bow shock, moving either toward or away from the neutron star. Flares are also produced by the simulations up to a level of $10^{37} \mathrm{erg} / \mathrm{s}$.

Conclusions. We have qualitatively reproduced the hard X-ray variations observed in Vela X-1 with hydrodynamic instabilities predicted by a simple model. More sophisticated phenomena, such as clumpy winds or the magnetic gating mechanism, are not excluded, but are not required either to explain the basic phenomenology.
\end{abstract}

Key words. X-rays: binaries - accretion, accretion disks - hydrodynamics - stars: winds, outflows - X-rays: individuals: Vela X-1

\section{Introduction}

Neutron stars or black-holes in high-mass X-ray binaries (HMXBs) accrete gas from the stellar wind of their massive OB-type stellar companions. A fraction of the gravitational potential energy is converted into X-rays, which ionizes and heats the surrounding gas. The X-ray emission can be used to investigate the structure of the stellar wind in situ (Walter \& Zurita Heras 2007).

Vela X-1 (=4U 0900-40) is a classical persistent and eclipsing super-giant high mass X-ray binary (sgHMXB). The system consists of an evolved B $0.5 \mathrm{Ib}$ supergiant (HD 77581) and of a massive neutron star $\left(M_{\mathrm{NS}}=1.86 M_{\odot}\right.$; Quaintrell et al. 2003). The neutron star orbits its massive companion with a period of about 8.9 days on a circular orbit $(e \approx 0.09$; Bildsten et al. 1997) with a radius of $\alpha=1.76 R_{*}$. The stellar wind is characterized by a mass-loss rate of $\sim 4 \times 10^{-6} M_{\odot} \mathrm{yr}^{-1}$ (Nagase et al. 1986) and a wind terminal velocity of $v_{\infty} \approx 1700 \mathrm{~km} \mathrm{~s}^{-1}$ (Dupree et al. 1980). The X-ray luminosity is typically $\sim 4 \times 10^{36} \mathrm{erg} \mathrm{s}^{-1}$, although high variability can be observed (Kreykenbohm et al. 1999).

Recent studies on the hard X-ray variability of Vela X-1 have revealed a rich phenomenology including flares and short off-states (Kreykenbohm et al. 2008). Both flaring activity and off-states were interpreted as the effect of a strongly structured wind. Fürst et al. (2010) characterized the X-ray variability of Vela X-1 with a log-normal distribution, interpreted in the context of a clumpy stellar wind. Off-states have been interpreted (Kreykenbohm et al. 2008) as an evidence for the propeller effect
(Illarionov \& Sunyaev 1975), possibly accompanied by leakage through the magnetosphere (Doroshenko et al. 2011). The quasispherical subsonic accretion model (Shakura et al. 2012, 2013) is an alternative, predicting that the repeatedly observed off-states in Vela X-1 are the result of a transition from Compton to radiative cooling (higher and lower luminosity, respectively).

In this paper we present new results from 2D hydrodynamic simulations of Vela X-1 and conclude that the observed phenomenology can be explained qualitatively without intrinsic clumping or the propeller effect. The code and simulations are described in Sect. 2. The simulation results are presented and compared to observations in Sect. 3 and are discussed in Sects. 4 and 5 .

\section{Hydrodynamic simulations}

\subsection{Hydrodynamic code}

The motion of the fluid is described by the Euler equations assuming mass, momentum, and energy conservation. The internal energy in each cell is described by the first law of thermodynamics. The following set of equations are therefore solved in a fixed, non-uniform mesh:

$\partial_{t} \rho+\nabla \cdot(\rho \boldsymbol{u})=0$

$\partial_{t}(\rho \boldsymbol{u})+\nabla \cdot(\rho \boldsymbol{u} \boldsymbol{u})+\nabla P=\boldsymbol{F}$,

and

$\partial_{t} E+\nabla \cdot(E \boldsymbol{u}+P \boldsymbol{u})=\boldsymbol{u} \cdot \boldsymbol{F}$, 
Table 1. Simulation parameters.

\begin{tabular}{ll}
\hline \hline Parameter & Value \\
\hline Donor star parameters & \\
$M_{*}$ & $23.1 M_{\odot}$ \\
$R_{*}$ & $30 R_{\odot}$ \\
$L_{*}$ & $2.5 \times 10^{5} L_{\odot}$ \\
$T_{*}$ & $40000 \mathrm{~K}$ \\
\hline Binary parameters & \\
$M_{\mathrm{NS}}$ & $1.86 M_{\odot}$ \\
$\alpha$ & $1.76 R_{*}$ \\
$L_{\mathrm{X}}$ & $4 \times 10^{36} \mathrm{erg} \mathrm{s}^{-1}$ \\
\hline CAK parameters & \\
CAK- $\alpha$ & 0.58 \\
CAK- $k$ & 0.80 \\
$\rho_{0}$ & $10^{-11} \mathrm{~g} \mathrm{~cm}^{-3}$ \\
\hline Wind parameters & \\
$\dot{M}_{\mathrm{W}}$ & $4 \times 10^{-6} M_{\odot} \mathrm{yr}^{-1}$ \\
$v_{\infty}$ & $1700 \mathrm{~km} \mathrm{~s}^{-1}$ \\
\hline
\end{tabular}

where we used $\partial_{t}=\partial / \partial t$. The primary variables of the simulations (the mass density $\rho$, gas pressure $P$, and fluid velocity $\boldsymbol{u}$ ) are completed by the total energy $E=\frac{1}{2} \rho \boldsymbol{u}^{2}+\rho e$ and the force $\boldsymbol{F}$, accounting for the Roche potential and the line-driven force (see Sect. 2.2). The specific internal energy $(e)$ is related to the pressure through the equation of state, $P=\rho e(\gamma-1)$, where $\gamma=5 / 3$ is the ratio of specific heats.

The VH- $1^{1}$ hydrodynamic code is described in detail in Blondin et al. (1990, 1991). The simulations of Vela X-1 take into account the gravity of the primary and of the neutron star, the radiative acceleration (Castor et al. 1975, CAK hereafter) of the stellar wind of the donor star, and the suppression of the stellar wind acceleration due to high ionization within the Strömgren sphere of the neutron star. The parameters are listed in Table 1.

The equations are solved in the orbital plane of the corotating reference system, where the lateral extent ( $\theta$ component) of the spherical mesh is only one cell. We also assumed a circular orbit and a synchronous rotation. The code uses the piecewise parabolic method for shock hydrodynamics developed by Colella \& Woodward (1984).

A computational mesh of 900 radial by 347 angular zones, extending from 1 to $\sim 25 R_{*}$ and in angle from $-\pi$ to $+\pi$ was employed. The grid is built in a non-uniform way to allow for higher resolution (up to $\sim 10^{9} \mathrm{~cm}$ ) toward the neutron star and is centered on the center of mass.

The initial wind density, velocity, pressure, and CAK parameters of the $2 \mathrm{D}$ simulartion are set by the results of a $1 \mathrm{D}$ CAK/Sobolev simulation (also extending up to $25 R_{*}$ ) of a single star and result in the standard $\beta \approx 0.8$ velocity law. In the binary potential, the wind takes a few days of simulation to relax and find a new equilibrium. The first three days of the simulations are therefore excluded from the variability analysis.

The wind reaching the radial outermost part of the mesh is characterized as an outflow boundary condition, meaning that it leaves the simulation domain. We also assume that the wind entering the cell that represents the surface of the neutron star is completely accreted, leaving an (almost) zero density and pressure. The matter falling from the surrounding cells is therefore in free fall (Hunt 1971; Blondin \& Pope 2009).

\footnotetext{
http://wonka.physics.ncsu.edu/pub/VH-1/
}

The time step of the simulations is $\sim 1 / 10 \mathrm{~s}$ and the variables of each cell are stored for each step. The code also calculates the ionization parameter $\left(\xi=L_{\mathrm{X}} / n r_{\mathrm{ns}}^{2}\right.$, where $n$ is the number density at the distance $r_{\mathrm{ns}}$ from the neutron star; Tarter et al. 1969) and the instantaneous mass and angular momentum accreted $\left(\dot{M}_{\text {acc }}\right)$ on the neutron star. The code was run for about 30 days, that is, more than three orbits. This is enough for the wind to reach a stable configuration and to study its short time-scale variability.

\subsection{Stellar wind acceleration}

The winds of hot massive stars are characterized observationally by the wind terminal velocity and the mass-loss rate. The velocity is described by the $\beta$-velocity law, $v=v_{\infty}\left(1-R_{*} / r\right)^{\beta}$, where $v_{\infty}$ is the terminal velocity and $\beta$ is the gradient of the velocity field. For supergiant stars, values for wind terminal velocities and mass-loss rates are in the range $v_{\infty} \sim 1500-3000 \mathrm{~km} \mathrm{~s}^{-1}$ and $\dot{M}_{\mathrm{w}} \sim 10^{-(6-7)} M_{\odot} \mathrm{yr}^{-1}$, respectively (Kudritzki \& Puls 2000).

The stellar winds of massive supergiant stars are radiatively driven by absorbing ultraviolet photons from the underlying photosphere. To properly simulate the radiation force driving the stellar wind, we used the CAK/Sobolev approximation,

$F_{\text {rad }}=\frac{\sigma_{\mathrm{e}} L_{*}}{4 \pi c R^{2}} k K_{\mathrm{FDC}}\left(\frac{1}{\sigma_{\mathrm{e}} \rho u_{\mathrm{th}}} \frac{\mathrm{d} u}{\mathrm{~d} R}\right)^{\alpha}$,

where $L_{*}$ is the stellar luminosity, $\sigma_{\mathrm{e}}$ is the electron scattering coefficient $\left(\approx 0.33 \mathrm{~cm}^{2} \mathrm{~g}^{-1}\right)$ and $u_{\text {th }}$ is the thermal velocity of the gas. The parameters CAK- $k$ and CAK- $\alpha$ are constants and correspond to the number and strength of the absorption lines, respectively (Castor et al. 1975). The effect of finite-disk correction (Friend \& Abbott 1986) has been accounted through the factor

$K_{\mathrm{FDC}}=\frac{(1+\sigma)^{1+\alpha}-\left(1+\sigma \mu^{2}\right)^{1+\alpha}}{(1+\alpha)\left(1-\mu^{2}\right) \sigma(1+\sigma)^{\alpha}}$,

where $\sigma=\frac{\mathrm{d} \ln u}{\operatorname{dn} R}-1$ and $\mu=\left(1-\frac{R_{*}^{2}}{R^{2}}\right)^{1 / 2}$, where $R$ and $u$ are the radial distance and velocity, respectively. The finite disk correction produces a shallow $\beta \approx 0.8$ velocity law, corresponding to the observations, rather than a steeper $\beta \approx 0.5$. The wind parameters, including the density at the bottom of the donor star atmosphere $\left(\rho_{0}\right)$, and the resulting mass-loss rate and wind terminal velocity are listed in Table 1 .

The radiation force is known to be unstable (Owocki \& Rybicki 1984), generating inhomogeneities and clumps (Owocki et al. 1988; Sundqvist \& Owocki 2013). In a close binary system the neutron star is a driver of the hydrodynamics, and models combining binarity and intrinsic instabilities are still to be developed.

The radiative acceleration of the wind is suppressed in case of X-ray photo-ionization. The effects of the X-ray radiation on the radiative acceleration force are complicated due to the large number of ions and line transitions that contribute to the opacity (Abbott 1982; Stevens \& Kallman 1990). Detailed NLTE wind models of the envelope of Vela X-1 show a photoionized bubble around the neutron star filled with stagnating flow (Krtička et al. 2012).

Assuming that the gas is in ionization equilibrium, the ionization state can be estimated with the $\xi$ parameter (Fransson \& Fabian 1980; Blondin et al. 1990). In our simulations we defined a critical ionization parameter $\left(\xi>10^{2.5} \mathrm{erg} \mathrm{cm} \mathrm{s}^{-1}\right)$ above 


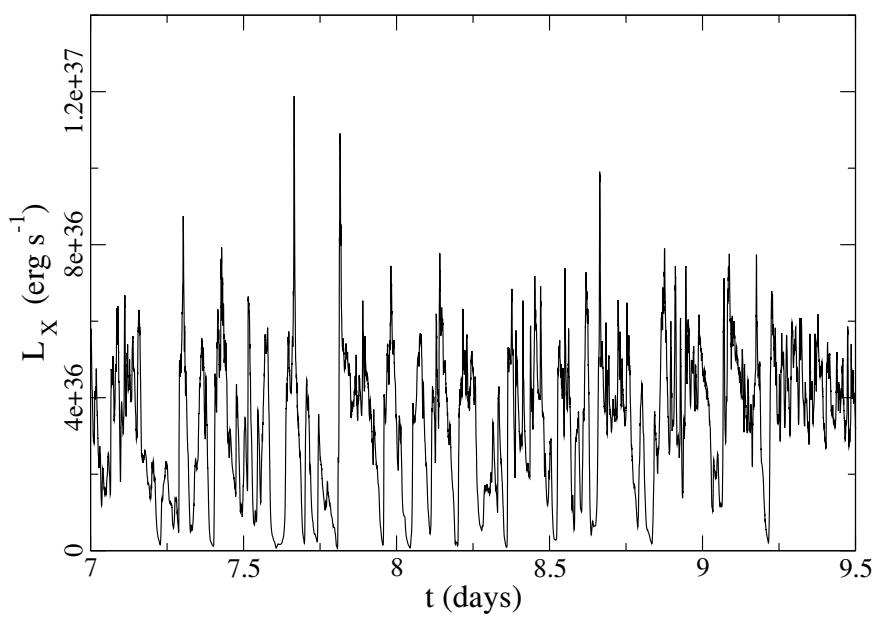

Fig. 1. Fraction of the simulated light curve, spanning 2.5 days (about $30 \%$ of an orbit).

which most of the elements responsible for the wind acceleration (e.g., C, N, O) are fully ionized (Kallman \& McCray 1982) and the radiative force becomes negligible $\left(F_{\text {rad }}=0\right.$ in Eq. (4)).

The main effect of the ionization is the reduction of the wind velocity in the vicinity of the neutron star and therefore the enhancement of the mass accretion rate onto the compact object (further increasing the effect). The outcomes of our simulations are not significantly affected by small variations $(\sim 20 \%)$ of the critical ionization parameter. However, larger variations (order of magnitude) significantly change the hydrodynamics. The suppression of the acceleration also triggers the formation of a dense wake at the rim of the Strömgren zone (Fransson \& Fabian 1980) and affects the absorption at late orbital phases. X-ray ionization can also affect the thermal state of the wind through X-ray heating and radiative cooling. Such effects are not included in our simulations.

\section{Results}

The observed hard X-ray light-curves of Vela X-1 were obtained form the INTEGRAL (Winkler et al. 2003) soft $\gamma$-ray imager ISGRI (Lebrun et al. 2003) in the 20-60 keV energy band and from the PCA (Jahoda et al. 2006) detector onboard RXTE in the 10-50 keV energy band (lower energies were excluded to avoid variable absorption effects). The light curves were obtained using the interface HEAVENS ${ }^{2}$ (Walter et al. 2010). The temporal resolutions of the ISGRI and PCA light curves are $\sim 40 \mathrm{~min}$ and $1 \mathrm{~min}$, respectively. We excluded data obtained during eclipses, using the orbital solution derived from Kreykenbohm et al. (2008).

The simulated X-ray light curve was obtained from the instantaneous mass-accretion rate $\left(L_{\mathrm{acc}}=\eta \dot{M}_{\mathrm{acc}} c^{2}\right)$, using a radiative efficiency of $\eta \approx 0.1$. Figure 1 shows a fraction of the simulated light curve. A number of off-states can be observed as well as flares reaching $\gtrsim 10^{37} \mathrm{erg} \mathrm{s}^{-1}$. Figure 3 shows a zoom on one of the off-states, where the light curve has been convolved with a sinusoidal of period of $283 \mathrm{~s}$ to account for the spin of the Vela X-1 pulsar. The properties of the simulated light curve and its comparison with the observations are described in the next subsections.

\footnotetext{
2 http://www.isdc.unige.ch/heavens
}

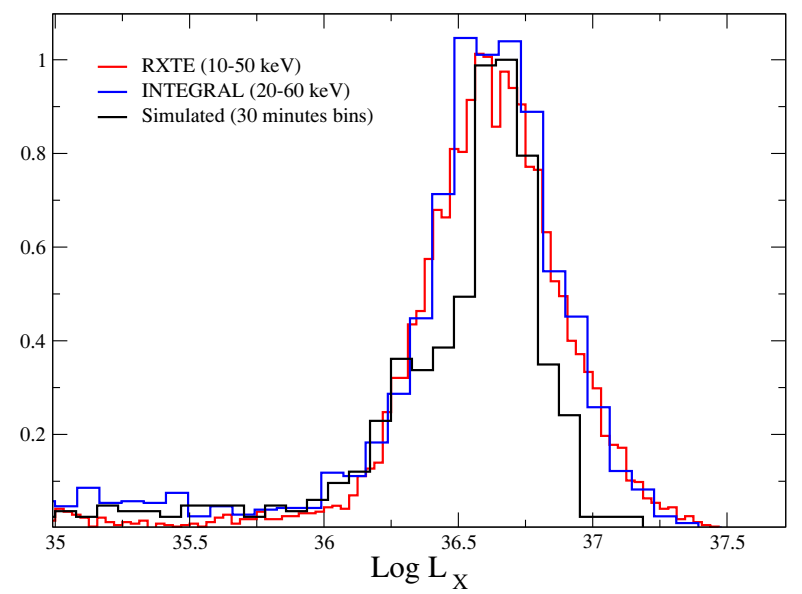

Fig. 2. X-ray luminosity distribution of Vela X-1 from RXTE (10-50 keV; red) and INTEGRAL (20-60 keV; blue) observations together with the distribution derived from the simulations (black). The simulated light curve was rebinned to a 30 -min bin size to match the INTEGRAL data.

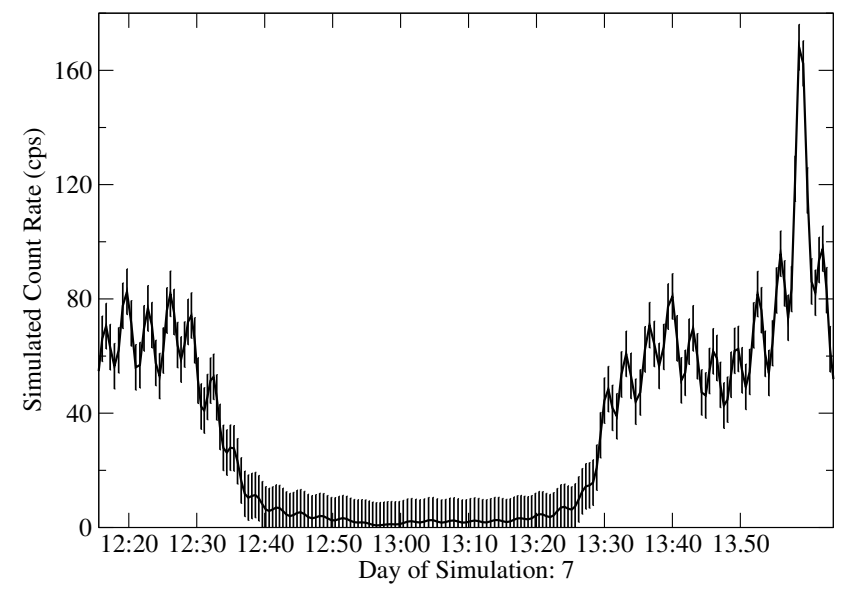

Fig. 3. Portion of the simulated light curve with $20 \mathrm{~s}$ time bins. The average luminosity of $\sim 4 \times 10^{36} \mathrm{erg} \mathrm{s}^{-1}$ corresponds to $\sim 70 \mathrm{cps}$. This figure can be directly compared to Fig. 6 in Kreykenbohm et al. (2008).

\subsection{Luminosity distributions}

We constructed histograms of the observed and simulated luminosities using the complete sample available (excluding the first three days of the simulations and the eclipses from the observations). The observed luminosity were derived from the X-ray count rates and responses assuming a distance of $1.9 \mathrm{kpc}$ (Nagase 1989), while $\eta$ was adjusted to the value of 0.107 to match the averaged luminosity of the simulation to the observed ones. The histograms are characterized by a peak at $\sim 4 \times$ $10^{36} \mathrm{erg} \mathrm{s}^{-1}$ (Fig. 2) and normalized to the same maximum amplitude for comparison.

The two histograms derived from the observations are very similar and shaped as a log-normal distribution with a lowluminosity tail. The log-normal standard deviation is $\sigma \approx 0.23$. The histogram of the 30-min binned simulated light curve is characterized by a narrower distribution $(\sigma=0.18)$, but more realistic simulations (e.g., including 3D, heating, cooling, and clumping effects) may generate more turbulence and match the observations better. Note that the histogram of the unbinned simulated light curve shows increased excess (by 25\%) at low luminosity $\left(\log L_{X} \sim 35.5\right)$. 

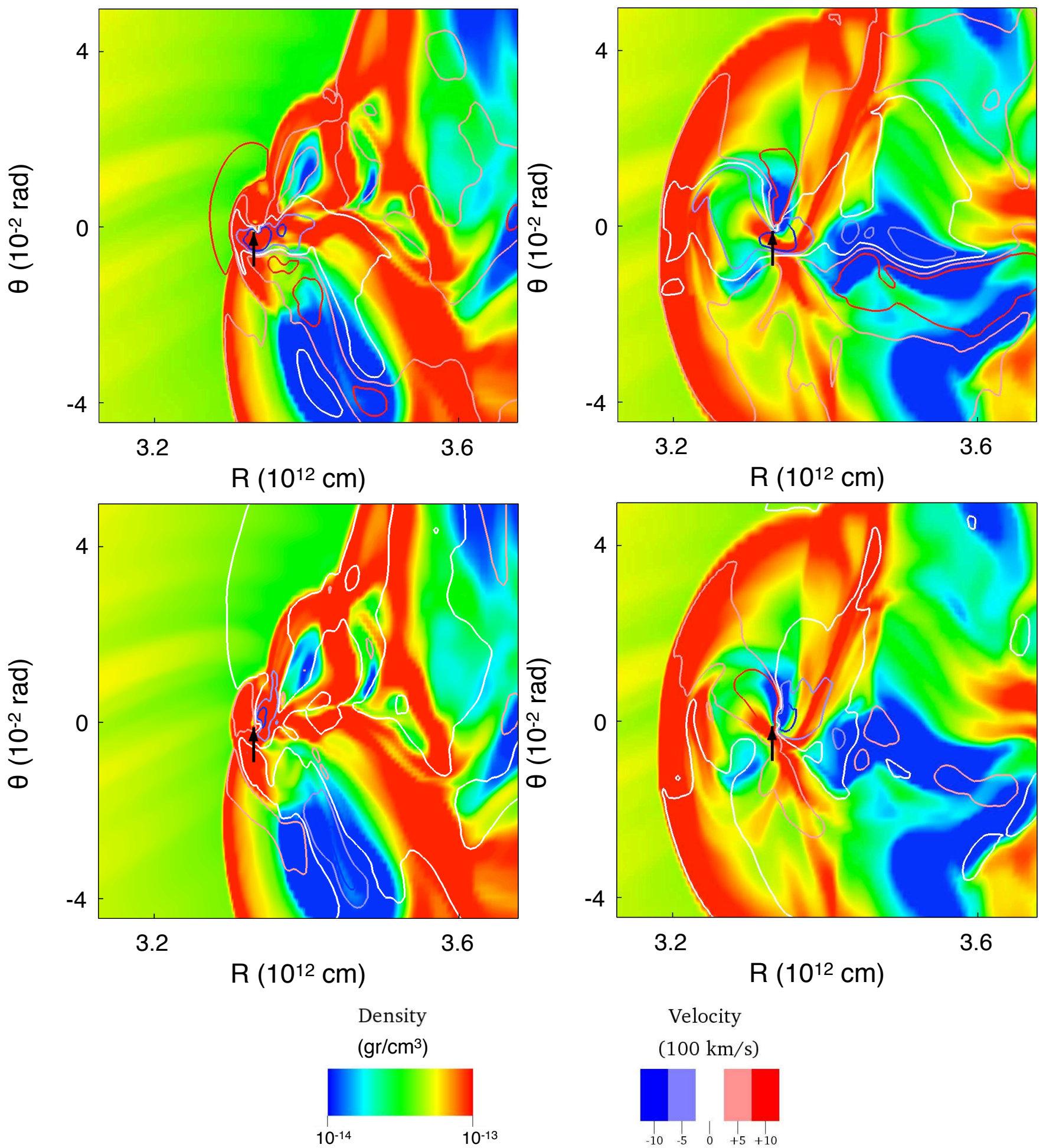

Velocity

$(100 \mathrm{~km} / \mathrm{s})$

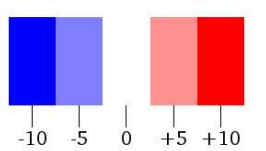

Fig. 4. Density distribution (in $\mathrm{gr}^{-3} \mathrm{~cm}^{-3}$ ) before (left columns) and during (right columns) the off-state. The upper and lower panels show the radial and angular velocity contours. The position of the neutron star is indicated by the black arrow.

\subsection{Off-states}

The simulated light curves feature many off-states. We defined an off-state as the state when the instantaneous X-ray luminosity dropped below $1 / 10$ of the average luminosity, $L_{\text {off }} \lessgtr$ $4 \times 10^{35} \mathrm{erg} \mathrm{s}^{-1} \approx 0.1\left\langle L_{\mathrm{X}}\right\rangle$, which is approximately the sensitivity limit of ISGRI in $20 \mathrm{~s}$.

Figure 4 shows the density maps and velocity contours before and during an off-state. The velocity contours are shown for the radial and angular velocities (upper and lower panels). As the bow shock on the left of the neutron star expands, the massaccretion rate decreases and an off-state occurs.

The typical size of the bubble sustained by the shock is of about $10^{11} \mathrm{~cm}$. Inside, the density drops at least by a factor of $\sim 10$ compared with the time-averaged density. This reduced density leads to the chop of the X-ray emission. The duration of the off-state varies with the size of the bubble.

Figure 5 shows the distribution of the off-state durations in the simulated light curve. The typical duration of most of the 


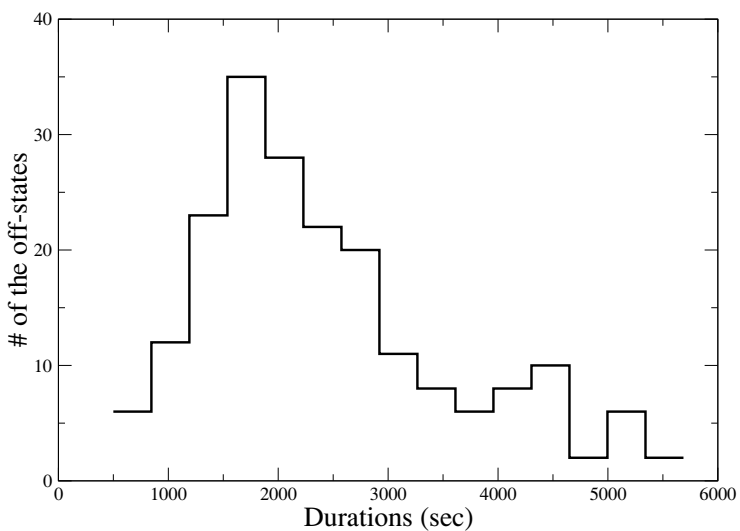

Fig. 5. Histogram of the duration of the off-states in the complete simulated light curve.

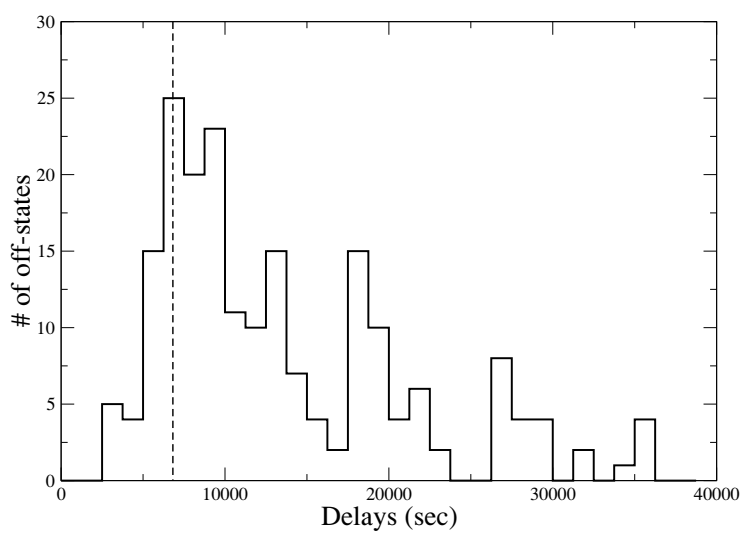

Fig. 6. Histogram of delay between two subsequent off-states using all the available simulated data. A total of 201 off-states have been identified. The dashed vertical line indicates the $6800 \mathrm{~s}$ quasi-periodicity detected in the observations.

off-states is about $30 \mathrm{~min}$ and ranges from $10 \mathrm{~min}$ to about two hours. Although there are few observed off-states, all of them last for between 5 and $30 \mathrm{~min}$, which coincides well with the simulations.

\subsection{Flaring activity}

In addition to the off-states, we identified prominent flares during the simulations. The flares reached luminosities of up to $L_{\text {flare }} \gtrsim$ $10^{37} \mathrm{erg} \mathrm{s}^{-1}$ for a duration of $\sim 5-30 \mathrm{~min}$. Figure 3 shows the brightest flare reaching $\sim 1.2 \times 10^{37} \mathrm{erg} \mathrm{s}^{-1} \approx 3\left\langle L_{\mathrm{X}}\right\rangle$ and lasting for $\sim 25 \mathrm{~min}$. The energy released during this flare is $\sim 10^{39} \mathrm{erg}$. These flares are compatible, in terms of dynamical range, with the flares of Vela X-1 observed by INTEGRAL (Kreykenbohm et al. 2008), but they are usually shorter, which explains why the luminosity distribution is narrower (see Fig. 2). We identified eight such flares $\left(L_{\mathrm{X}}>10^{37} \mathrm{erg} \mathrm{s}^{-1}\right)$ in the 30-day simulation, while Kreykenbohm et al. (2008) detected five flares during an observation of about two weeks.

\subsection{Quasi-periodicity}

Although the simulated light curve lacks any periodic signal, we can identify an almost quasi-periodic behavior related to the spacing of the off-states. Figure 6 shows the histogram of the time intervals between successive off-states. The distribution peaks in the range 6500-7000 s, close to the transient

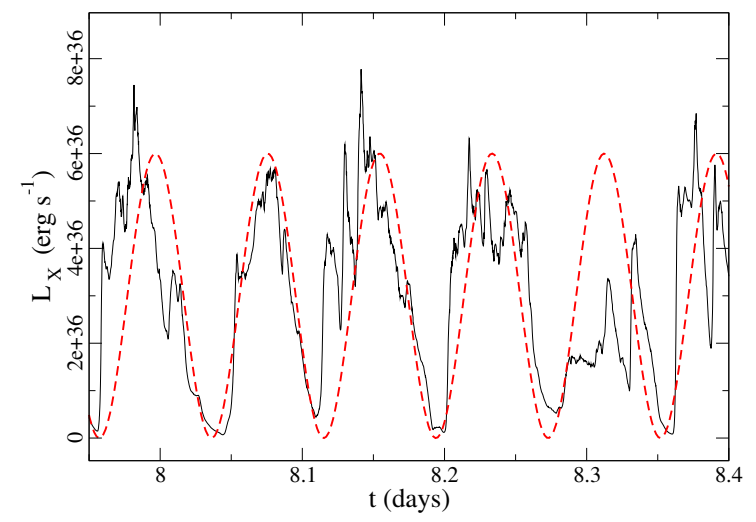

Fig. 7. Section of the simulated light curves of Vela X-1 together with a sine wave with a period of $6820 \mathrm{~s}$ (red dashed line).

period of $\sim 6800 \mathrm{~s}$ detected with INTEGRAL by Kreykenbohm et al. (2008) during about ten hours. The off-states labeled 1, 2, 3, and 5 in Kreykenbohm et al. (2008), which reach less than $10 \mathrm{ct} / \mathrm{s}$, are within $\sim 0.1$ in phase of the minima of the extension of the modulation mentioned earlier. It is therefore plausible that the observed off-states and modulations are the signature of a single physical mechanism driving the variability. In our simulations, these modulations last typically for 8-16 h and repeat every few days. Some signal is also detected at multiples of that period, suggesting that the density of the bubbles does not always reach the threshold we have defined for an off-state. A section of the simulated light curve featuring a very good coherence with the transient period of $6820 \mathrm{~s}$ is shown in Fig. 7.

\section{Discussion}

We have simulated the accretion flow in Vela X-1 with high spatial and temporal resolutions around the neutron star and found flares and off-states that qualitatively agree with the observed ones. In particular, off-states are regularly produced and correspond to an instability of the bow shock surrounding the neutron star.

In wind-fed HMXB systems (Taam et al. 1988) the accretion flows are complex and characterized by episodic accretion (Soker 1990, 1991) with an accreted angular momentum varying in direction and close to zero, on average. This is known as the "flip-flop" instability (e.g., Matsuda et al. 1987; Taam \& Fryxell 1988), which is interpreted by the formation of transient accretion disks (Blondin \& Pope 2009). We also observed that the accreted angular momentum changes sign regularly in our simulations between flares and off-states, but we cannot characterize them with the usual "flip-flop" instability because the characteristic duration of the variability is much shorter in our case and is not linked to the formation of accretion disks.

Our simulations predict the formation of low-density bubbles behind the bow shock, around the neutron star, resulting in the X-ray off-states. These bubbles are about ten times larger than the Bondi-Hoyle-Lyttleton (BHL; see e.g., Edgar 2004) accretion radius $\left(\sim 10^{10} \mathrm{~cm}\right)$. The bow shock is unstable, it appears close to the neutron star, and moves away up to a distance $\left(\gtrsim 10^{11} \mathrm{~cm}\right.$; see right panel of Fig. 4) before gradually falling back when a stream of gas eventually reaches the neutron star and produces a new rise of the X-ray flux. The accretion stream can either move toward the left or the right.

This "breathing" behavior is neither perfectly periodic nor continuous, but can be observed most of the time in the simulation and is at the origin of the off-states. Because the internal 
energy (pressure) of the low-density bubble is a fraction $(\sim 1 / 10)$ of the gravitational potential at the bow shock, the modulation period ( $\sim 6500-7000 \mathrm{~s})$ is similar to the free-fall time $(\sim 2000 \mathrm{~s})$ at this position. Neither the free-fall time from the BHL accretion radius $\left(t_{\mathrm{ff}} \sim 2 \mathrm{~min}\right)$ nor that from the magnetospheric radius $\left(t_{\mathrm{ff}} \sim 3 \mathrm{~s}\right)$ are consistent with the observed modulation time-scale.

Modulations are also produced by recent idealized 3D BHL accretion simulations (Blondin \& Raymer 2012), although weaker than in the 2D case (Ruffert 1994, 1997, 1999). The timescale of these modulations is related to the accretion radius, and their amplitude is weaker than what we are observing.

Krumholz et al. (2006) showed that the Bondi-Hoyle accretion of supersonic turbulent gas has a much higher density than the simple BHL formulation. They obtained a log-normal distribution of the mass-accretion rate, but it is much wider than what we found. The source of the turbulence is completely different in their case. The simulated log-normal distribution features more low-luminosity excess than observed. Passot \& Vázquez-Semadeni (1998) found similar excesses in 1D simulations of highly compressible gas. It is unclear whether the additional excesses have a physical meaning.

Reality is certainly more complex, indeed, massive stars are known to have clumpy winds (Owocki et al. 1988; Dessart \& Owocki 2003; Oskinova et al. 2006; Sundqvist \& Owocki 2013), and a rich phenomenology might be produced (Walter \& Zurita Heras 2007). Clumpy-wind scenarios can enhance the instabilities and reduce the X-ray luminosity for extended periods of time in the class of super-giant fast X-ray transients (SFXT, Negueruela et al. 2006). One-dimensional studies of the influence of strong density and velocity fluctuations of the wind resulted in episodic X-ray variability, which too large, however, to describe the observations (Oskinova et al. 2012). The lack of multiscale and multidimensional hydrodynamic simulations of a macroscopic clumpy wind in a binary system does not yet allow understanding the interplay between intrinsic clumping and the various effects of the neutron star.

\section{Conclusion}

We compared hard X-ray light curves of Vela X-1 obtained with RXTE and INTEGRAL with the predictions of hydrodynamic simulations.

The simulated light curve is highly variable (see Fig. 1), as observed (Kreykenbohm et al. 2008). The dynamical range of variability is on the order of $\sim 10^{3}$ between off-states and the brightest flares. The X-ray luminosity is a direct probe of the instantaneous mass-accretion rate and of the density fluctuations around the neutron star. The duration of the off-states in our simulation ( 1 to $5 \mathrm{ks}$ ) corresponds to the free-fall time of low-density bubbles building up behind the bow shock that surrounds the neutron star.

The log-normal flux distribution resulting from the observations and from the simulation is a characteristic result of selforganised criticality (Bak et al. 1988; Crow \& Shimizu 1988; Uttley et al. 2005). In our case, the criticality condition is probably related to the direction of the bow shock and accretion stream that can lead or trail the neutron star. Oscillations between these positions lead to the succession of off-states, flares, and more generally to the near- log-normal flux distribution. The flares correspond to accretion of a mass of $\sim 10^{19} \mathrm{gr}$, much lower than inferred for the clumps in the case of SFXTs, because the variability is driven by small-scale instabilities in Vela X-1.
Our hydrodynamic simulations are sufficient to explain the observed behavior without the need for intrinsically clumpy stellar wind or high magnetic fields and gating mechanisms. More advanced and realistic simulations that include such phenomena are needed to understand their interplay with the hydrodynamic effects of the neutron star and to reveal the full accretion phenomenology in classical sgHMXBs.

Acknowledgements. A.M. would like to thank J. Blondin for fruitful discussions and hospitality at the NCSU, as well as acknowledge support by the Polish NCN grant 2012/04/M/ST9/00780.

\section{References}

Abbott, D. C. 1982 , ApJ, 259, 282

Bak, P., Tang, C., \& Wiesenfeld, K. 1988, Phys. Rev. A, 38, 364

Bildsten, L., Chakrabarty, D., Chiu, J., et al. 1997, ApJS, 113, 367

Blondin, J. M., \& Pope, T. C. 2009, ApJ, 700, 95

Blondin, J. M., \& Raymer, E. 2012, ApJ, 752, 30

Blondin, J. M., Kallman, T. R., Fryxell, B. A., \& Taam, R. E. 1990, ApJ, 356, 591

Blondin, J. M., Stevens, I. R., \& Kallman, T. R. 1991, ApJ, 371, 684

Castor, J. I., Abbott, D. C., \& Klein, R. I. 1975, ApJ, 195, 157

Colella, P., \& Woodward, P. R. 1984, J. Comput. Phys., 54, 174

Crow, E. L., \& Shimizu, K. 1988, Lognormal Distributions: Theory and Applications (New York: Dekker)

Dessart, L., \& Owocki, S. P. 2003, A\&A, 406, L1

Doroshenko, V., Santangelo, A., \& Suleimanov, V. 2011, A\&A, 529, A52

Dupree, A. K., Gursky, H., Black, J. H., et al. 1980, ApJ, 238, 969

Edgar, R. 2004, New Astron. Rev., 48, 843

Fransson, C., \& Fabian, A. C. 1980, A\&A, 87, 102

Friend, D. B., \& Abbott, D. C. 1986, ApJ, 311, 701

Fürst, F., Kreykenbohm, I., Pottschmidt, K., et al. 2010, A\&A, 519, A37

Hunt, R. 1971, MNRAS, 154, 141

Illarionov, A. F., \& Sunyaev, R. A. 1975, A\&A, 39, 185

Jahoda, K., Markwardt, C. B., Radeva, Y., et al. 2006, ApJS, 163, 401

Kallman, T. R. \& McCray, R. 1982, ApJS, 50, 263

Kreykenbohm, I., Kretschmar, P., Wilms, J., et al. 1999, A\&A, 341, 141

Kreykenbohm, I., Wilms, J., Kretschmar, P., et al. 2008, A\&A, 492, 511

Krtička, J., Kubát, J., \& Skalický, J. 2012, ApJ, 757, 162

Krumholz, M. R., McKee, C. F., \& Klein, R. I. 2006, ApJ, 638, 369

Kudritzki, R., \& Puls, J. 2000, ARA\&A, 38, 613

Lebrun, F., Leray, J. P., Lavocat, P., et al. 2003, A\&A, 411, L141

Matsuda, T., Inoue, M., \& Sawada, K. 1987, MNRAS, 226, 785

Nagase, F. 1989, PASJ, 41, 1

Nagase, F., Hayakawa, S., Sato, N., Masai, K., \& Inoue, H. 1986, PASJ, 38, 547

Negueruela, I., Smith, D. M., Reig, P., Chaty, S., \& Torrejón, J. M. 2006, in The X-ray Universe 2005, ed. A. Wilson, ESA SP, 604, 165

Oskinova, L. M., Feldmeier, A., \& Hamann, W.-R. 2006, MNRAS, 372, 313

Oskinova, L. M., Feldmeier, A., \& Kretschmar, P. 2012, MNRAS, 421, 2820

Owocki, S. P., \& Rybicki, G. B. 1984, ApJ, 284, 337

Owocki, S. P., Castor, J. I., \& Rybicki, G. B. 1988, ApJ, 335, 914

Passot, T., \& Vázquez-Semadeni, E. 1998, Phys. Rev. E, 58, 4501

Quaintrell, H., Norton, A. J., Ash, T. D. C., et al. 2003, A\&A, 401, 313

Ruffert, M. 1994, ApJ, 427, 342

Ruffert, M. 1997, A\&A, 317, 793

Ruffert, M. 1999, A\&A, 346, 861

Shakura, N., Postnov, K., Kochetkova, A., \& Hjalmarsdotter, L. 2012, MNRAS, 420, 216

Shakura, N., Postnov, K., \& Hjalmarsdotter, L. 2013, MNRAS, 428, 670

Soker, N. 1990, ApJ, 358, 545

Soker, N. 1991, ApJ, 376, 750

Stevens, I. R. \& Kallman, T. R. 1990, ApJ, 365, 321

Sundqvist, J. O., \& Owocki, S. P. 2013, MNRAS, 428, 1837

Taam, R. E., \& Fryxell, B. A. 1988, ApJ, 327, L73

Taam, R. E., Brown, D. A. \& Fryxell, B. A. 1988, ApJ, 331, L117

Tarter, C. B., Tucker, W. H., \& Salpeter, E. E. 1969, ApJ, 156, 943

Uttley, P., McHardy, I. M., \& Vaughan, S. 2005, MNRAS, 359, 345

Walter, R., \& Zurita Heras, J. 2007, A\&A, 476, 335

Walter, R., Rohlfs, R., Meharga, M. T., et al. 2010, in Proc. 8th INTEGRAL

Workshop The Restless Gamma-ray Universe (INTEGRAL 2010), 162

Winkler, C., Courvoisier, T., Di Cocco, G., et al. 2003, A\&A, 411, L1 